\title{
Effects of thrombocytopenia in pregnancy
}

\author{
Shital N. Kapadiya ${ }^{1}$, Harsh S. Patel ${ }^{1 *}$, Kartikey G. Parmar ${ }^{2}$
}

${ }^{1}$ Department of Obstetrics and Gynecology, ${ }^{2}$ Department of Medicine, B. J. Medical College, Ahmedabad, Gujarat, India

Received: 05 January 2018

Accepted: 31 January 2018

\section{* Correspondence:}

Dr. Harsh S. Patel,

E-mail: patel.harsh2502@gmail.com

Copyright: () the author(s), publisher and licensee Medip Academy. This is an open-access article distributed under the terms of the Creative Commons Attribution Non-Commercial License, which permits unrestricted non-commercial use, distribution, and reproduction in any medium, provided the original work is properly cited.

\section{ABSTRACT}

Background: Thrombocytopenia defined as platelet count of less than 1,50,000/cu.mm. Thrombocytopenia is divided according to severity into mild moderate and severe types. Multiple factors are responsible.

Methods: This is a retrospective study of one-year period including 120 pregnant patients irrespective of their gestational age at civil hospital Ahmedabad. Etiology of this condition are identified and analyzed.

Results: Gestational Thrombocytopenia is the most common etiology. This condition is self-limiting usually.

Conclusions: Platelet count estimation should be a routine at first antenatal visit for timely diagnosis and to achieve favorable fetomaternal outcome.

Keywords: Antenatal visit, Fetomaternal outcome, Gestational thrombocytopenia

\section{INTRODUCTION}

Thrombocytopenia defined as platelet count of less than $1,50,000 /$ cu.mm. It is a common hematological disorder. $^{1,2}$ It is second only to anemia as the most common hematological abnormality in pregnancy. ${ }^{3}$ Thrombocytopenia is being more frequently diagnosed in pregnant women since the last 20 years. Thrombocytopenia in pregnant women may result from the effects of diverse processes, which may be either physiological or pathological. ${ }^{4}$

Thrombocytopenia is divided according to severity into mild $(100,000$ to 150,000$)$, moderate $(50,000$ to 100,000$)$ and severe (less than 50,000) thrombocytopenia ${ }^{4}$. The increased recognition of maternal and fetal thrombocytopenia has resulted in controversies regarding management. In fact, screening at all for thrombocytopenia is an issue, because the reason for this common condition usually is benign. Performing studies on both the mother and the fetus can cause considerable morbidity and mortality, and the mode of delivery, e.g., cesarean section rather than vaginal delivery, has not improved outcome.

Health care providers must weigh the benefits of interventions in relation to cost and morbidity against the risk of maternal and fetal bleeding complications. ${ }^{6}$

The majority of thrombocytopenic pregnant women is healthy, has no history of thrombocytopenia, and is incidentally diagnosed by blood testing. This condition, called incidental or gestational thrombocytopenia (GT), usually has no influence on pregnancy, labour and delivery or on the new born. ${ }^{1}$ There may not be a risk of severe hemorrhage in GT, but preeclampsia, HELLP syndrome and ITP (Immune thrombocytopenic purpura) expose mother and child to potentially life-threatening complications. ${ }^{5}$

Thrombocytopenia in pregnancy is divided according to etiology into gestational, medical (ITP, hypersplenism, hepatic disorders etc.) and obstetric (hypertensive disorders, DIC) thrombocytopenia. ${ }^{2}$ 
Aims and objectives are to determine the etiology, adverse effects and interventions required in relation to thrombocytopenia in pregnancy.

\section{METHODS}

The present study was a retrospective study. No. of patients were 120 obstetric patients at carries out at BJ Medical College and Civil Hospital, Ahmedabad, Gujarat, India.

\section{Exclusion criteria}

Patients with pancytopenia and bone marrow suppression were excluded from the study.

Records of obstetric patients were studied from Department of Obstetrics and Gynecology BJ Medical College Ahmedabad, from September 2016 to August 2017. Total 6830 obstetric patients were admitted in the Obstetrics and Gynaecology Department during this period and among them 120 patients (1.8\%) had thrombocytopenia along with pregnancy and these case records were included in the study. Patients were included irrespective of gestational age. All women had previous records of platelet count estimation. Platelet count assessment had been done through automated blood count analyzer with routine antenatal hematological evaluation of the patient.

All women had been subjected to blood test for $\mathrm{Hb}$, TLC, DLC, bleeding time, clotting time, RFT, LFT, HBsAg and HIV. Women with fever had been tested for dengue IgM. Coagulation tests (PT, APTT, FDP and fibrinogen) had been done in those with signs or symptoms of DIC. Platelet counts are repeated in our institute, once in each trimester and in the postpartum period at 1 and 6 weeks. Obstetrical examination along with obstetrical intervention was done when needed. Descriptive statistics were used, and percentages were calculated for qualitative variables like cause of thrombocytopenia, complications, maternal outcome. Babies of all cases had been tested for thrombocytopenia and were followed up for any complications.

\section{RESULTS}

In the present study, frequency of thrombocytopenia during pregnancy was $1.8 \%$. The present study found no influence of age and religion on prevalence of thrombocytopenia in pregnancy. The mean age of patients was $27( \pm 5.594)$ years. The youngest patient was 18 years old and oldest was 35 years old. The mean gestational age was $34( \pm 5.4)$ weeks. The parity status of these cases was multigravida $64(53.3 \%)$, primigravida $50(41.6 \%)$ and grand multipara $6(5.0 \%)$. Majority of the patients $78(65 \%)$ did not receive antenatal care. Thrombocytopenia diagnosed before pregnancy in 2 patients $(2.4 \%)$, during pregnancy in 74 patients $(61.66 \%)$, during labour in 44 patients $(36.66)$.
Table 1: Distribution of cases of thrombocytopenia according to etiology.

\begin{tabular}{|lll|}
\hline Cause & No of cases & Percentage \\
\hline Gestational & 77 & 64.16 \\
\hline Obstetrics & 26 & 21.67 \\
\hline $\begin{array}{l}\text { PIH and } \\
\text { Preeclampsia }\end{array}$ & 18 & 15 \\
\hline Eclampsia & 4 & 3 \\
\hline DIC & 4 & 3 \\
\hline Medical & 17 & 14.17 \\
\hline Hypersplenism & 3 & 2.5 \\
\hline Hepatic disorders & 2 & 1.67 \\
\hline Malaria & 7 & 5.83 \\
\hline Megaloblastic anemia & 3 & 2.5 \\
\hline ITP & 2 & 1.67 \\
\hline
\end{tabular}

Gestational thrombocytopenia was the most common cause around $64 \%$ of all thrombocytopenia in this study with a platelet count ranging from 65,000 to 1,35,000/cu.mm. Thrombocytopenia due to HELLP syndrome and severe preeclampsia were the second common cause around $22 \%$ of all thrombocytopenia in this study. Medical causes are responsible for thrombocytopenia in $14 \%$ of patients in this study. Total 101 patients delivered while 19 patients remain undelivered. Most of the patients (115) survived. In the present study five mortality was recorded in relation to thrombocytopenia, in which 3 due to HELLP syndrome and its complication, which was $60 \%$, other two due to $\mathrm{PPH}$, which was $40 \%$ of total mortality.

Table 2: Maternal outcome.

\begin{tabular}{|lll|}
\hline Variables & No. of cases & Percentage \\
\hline Delivered & 101 & 84.33 \\
\hline Undelivered & 19 & 15.66 \\
\hline Survived & 115 & 95.8 \\
\hline Died & 5 & 4.2 \\
\hline $\begin{array}{l}\text { Causes of maternal mortality } \\
\text { Post-partum hemorrhage }\end{array}$ & 2 & 40 \\
\hline $\begin{array}{l}\text { HELLP syndrome and } \\
\text { its complications }\end{array}$ & 3 & 60 \\
\hline
\end{tabular}

\section{DISCUSSION}

Thrombocytopenia is a common problem during pregnancy, often under diagnosed and mismanaged. Limited data was available from early pregnancy as a significant proportion of antenatal women in our scenario seek advice only during late pregnancy or when a complication arises. Thrombocytopenia defined as platelet count of less than 1,50,000/cu.mm. Sainio et al reported a prevalence of thrombocytopenia in a population-based surveillance study to be $7.3 \% .^{5}$ Thus, the prevalence of thrombocytopenia in Indian population is similar to world literature (5-12\%). Ajzenburg et al. assumed that gestational thrombocytopenia occurs due to increased platelet consumption within the placental 
circulation and/or normal inhibition of megakaryocytopoiesis. It followed a benign course without any adverse effect and need for intervention during pregnancy. The platelet count falls progressively during pregnancy and rarely drops below 70,000/Cu.mm. Women are asymptomatic and have a completely negative history of abnormal bleeding. There is no preconception history of thrombocytopenia, an early gestation or preconception platelet counts are normal, and the platelet counts returns to normal within 2 to 12 weeks postpartum. Gestational thrombocytopenia has no impact on either the mother or the fetus. The early gestation or preconception platelet count becomes extremely important when differentiating this disorder from idiopathic thrombocytopenia purpura, with which it is commonly confused.

Thrombocytopenia in pregnancy is caused due to HELLP syndrome and severe preeclampsia were the $2^{\text {nd }}$ common cause $(21.67 \%)$ in the present study. Majority of our patients were referred from secondary care hospitals who remained unbooked throughout the pregnancy and came in emergency in critical condition. Activation of both the coagulation and fibrinolytic systems leads to the development of severe thrombocytopenia and disseminated intravascular coagulation (DIC) which occurs in some patients with preeclampsia. We observed that HELLP syndrome caused hemolysis, altered liver functions, lowering of platelets and severe hypertension in our patients and was associated with high maternal and fetal morbidity and mortality due to placental abruption, preterm deliveries, low APGAR scores, intrauterine growth retardation, stillbirths and maternal deaths. ${ }^{5}$

ACOG recommended that the definitive treatment of maternal thrombocytopenia in the setting of PIH with HELLP syndrome is termination of pregnancy. Platelet transfusions are less effective in these women because of accelerated platelet destruction. 5 Two situations in which additional platelets should be given are:

- To treat severe thrombocytopenia with ongoing bleeding, and

- To increase the platelet count to more than $50,000 / \mathrm{L}$ for an operative delivery. ${ }^{7}$

The platelet count usually begins returning to normal within 72 hours of delivery. ${ }^{8,9}$

ITP affects only 1-2 of every 10,000 pregnancies. Shehata $\mathrm{N}$ et al Concluded that detection of thrombocytopenia prior to 28 weeks of gestation is predictive of ITP. ${ }^{1}$ We found 1 case of ITP in the present study, which was $1.2 \%$ of total thrombocytopenia cases, which was detected in $2^{\text {nd }}$ trimester. The diagnosis of ITP is based on exclusion and only be made if other causes have been excluded. When ITP appears during pregnancy, both mother and fetus can be affected as antiplatelet antibody IgG can cross the placenta and cause thrombocytopenia. It is difficult to predict which fetus will be affected. The best predictor of severe neonatal thrombocytopenia is a previously affected child. ${ }^{1}$ Corticosteroids is the first line therapy; law doses of steroid is safe and effective in pregnancy. ${ }^{1}$ Intravenous immunoglobulins may be used in women requiring prolong therapy or resistant to steroid therapy or require high maintenance dose. ${ }^{1}$ Platelet transfusion are given as last resort for bleeding or prior to surgery. Idiopathic thrombocytopenic purpura (ITP) was found in two patients, infants of both case survived and did not require any treatment.

Women with ITP and platelet counts over 50,000/cu.mm must be managed conservatively during labour and delivery and a caesarean section is performed only for obstetrical indications. ${ }^{1}$ Often one has to wait till the postpartum period to differentiate between gestational thrombocytopenia of pregnancy which, resolves itself following delivery or immune thrombocytopenia which does not subside after delivery. The challenge to the clinician is to weigh the risks of maternal and fetal bleeding complications against the benefits of diagnostic tests and interventions.

\section{CONCLUSION}

The baseline low platelet counts and declining trend with increasing gestational age predispose Indian women to increased risk of thrombocytopenia in pregnancy. Thus, platelet count estimation should be a routine at first antenatal visit for timely diagnosis and to achieve favourable fetomaternal outcome in all types of thrombocytopenia during pregnancy.

\section{Funding: No funding sources}

Conflict of interest: None declared

Ethical approval: The study was approved by the Institutional Ethics Committee

\section{REFERENCES}

1. Shehata N, Burrows R, Kelton JG. Gestational thrombocytopenia. Clin Obst Gynaecol. 1999;42(2):327-34.

2. Burrows RF, Kelton JG. Thrombocytopenia at delivery (a prospective survey of 6,715 deliveries). Am J Obstet Gynaecol. 1990;162:731-4.

3. Sullivan CA, Martin JN Jr. Management of the obstetric patients with thrombocytopenia. Clin Obstet Gynecol. 1995;38:521-34.

4. Magann EF, Martin JN. Twelve steps to optical management of HELLP syndrome. Mississippi and Tennessee classification systems for HELLP syndrome. Clin Obstet Gynecol. 1999;42(3):532-50.

5. Sainio S, Kekomaki R, Rikonen S, Teramo K. Maternal thrombocytopenia at term: a populationbased study. Acta Obstetr Gynecol Scand. 2000;79(9):744-9.

6. Levy JA, Murphy LD. Thrombocytopenia in pregnancy. JABFP. 2002;15(4):290-7. 
7. Silver R, Berkowitz R, Bussel J. Thrombocytopenia in pregnancy. Practice bulletin, No 6. Chicago: American College of Obstetrics and Gynecology, Chicago; 1999.

8. Padden MO. HELLP syndrome: recognition and perinatal management. Am Fam Physician. 1999;60:829-36.

9. Egerman RS, Sibai BM. HELLP syndrome. Clin Obstet Gynecol. 1999;42:381-9.

10. Mathews JH, Benjamin S, Gill DS, Smith NA. Pregnancy associated thrombocytopenia definition, incidence and natural history. Acta Hematol. 1990;84:24-9.

11. Boehlen F, Hohfeld P. Platelet count at term pregnancy: a reappraisal of the threshold. Obstet Gynecol. 2000;95(1):29-33.

12. Karim R, Sacher RA. Thrombocytopenia in pregnancy. Curr Hematol Resp. 2004;3(2):128-33.

Cite this article as: Kapadiya SN, Patel HS, Parmar KG. Effects of thrombocytopenia in pregnancy. Int $\mathbf{J}$ Reprod Contracept Obstet Gynecol 2018;7:1044-7. 